\title{
Outcomes of Endovascular Thrombectomy with and without Thrombolysis for Acute Large Artery Ischaemic Stroke at a Tertiary Stroke Centre
}

\author{
Chee-Keong Wee ${ }^{a}$ William McAuliffe ${ }^{b}$ Constantine C. Phatouros ${ }^{b}$ \\ Timothy J. Phillips ${ }^{b}$ David Blacker ${ }^{a}$ Tejinder P. Singh ${ }^{b} \quad$ Ellen Baker ${ }^{a}$ \\ Graeme J. Hankey a, c \\ a Department of Neurology, Sir Charles Gairdner Hospital, Perth, WA, Australia; \\ ${ }^{b}$ Neurological Intervention and Imaging Service of Western Australia (NIISWA), Perth, WA, \\ Australia; ' School of Medicine and Pharmacology, The University of Western Australia, \\ Perth, WA, Australia
}

\author{
Keywords \\ Large vessel occlusion · Endovascular thrombectomy · Ischaemic stroke
}

\begin{abstract}
Background and Purpose: Endovascular thrombectomy (EVT) improves the functional outcome when added to best medical therapy, including alteplase, in patients with acute ischaemic stroke secondary to large vessel occlusion (LVO) in the anterior circulation. However, the evidence for EVT in alteplase-ineligible patients is less compelling. It is also uncertain whether alteplase is necessary in patients with successful recanalization by EVT, as the treatment effect of EVT may be so powerful that bridging alteplase may not add to efficacy and may compromise safety by increasing bleeding risks. We aimed to survey the proportion of patients suitable for EVT who are alteplase-ineligible and to compare the safety and effectiveness of standard care of acute large artery ischaemic stroke by EVT plus thrombolysis with that of EVT alone in a tertiary hospital clinical stroke service. Methods: We performed a retrospective analysis of acute ischaemic stroke patients treated with EVT at our centre between October 2013 and April 2016, based on a registry with prospective and consecutive patient collection. Individual patient records were retrieved for review. Significant early neurological improvement was defined as a NIHSS score of $0-1$, or a decrease from baseline of $\leq 8$, at $24 \mathrm{~h}$ after stroke onset. Results: Fifty patients with acute ischaemic stroke secondary to LVO in the anterior circulation received EVT in this period, of whom 21 (42\%) received concurrent alteplase and 29 (58\%) EVT alone. The 2 groups had similar baseline characteristics and similar outcomes. Significant neurological improvement at $24 \mathrm{~h}$ occurred in $47.6 \%$ of the patients
\end{abstract}


with EVT and bridging alteplase and in $51.7 \%$ of the patients with EVT alone ( $p=0.774)$. Mortality during acute hospitalization was $20 \%$ for the bridging alteplase group versus $7.1 \%$ for EVT alone $(p=0.184)$. Intracranial haemorrhage rates were $14.3 \%$ for bridging alteplase versus $20.7 \%$ for EVT alone ( $p=0.716)$. Local complications, groin haematoma $(23.8$ vs. $10.3 \%)$ and groin pseudoaneurysms ( 4.8 vs. $0 \%)(p=0.170)$, were not significantly different. Conclusion: Our study highlights the relatively large proportion of patients suitable for EVT who have a contraindication to alteplase and raises the hypothesis that adding alteplase to successful EVT may not be necessary to optimize functional outcome. The results are consistent with observational data from other endovascular centres and support a randomised controlled trial of EVT versus EVT with bridging alteplase.

\section{Background and Purpose}

Endovascular thrombectomy (EVT) has been shown in 7 recent randomised trials to improve functional outcome when added to best medical therapy in patients with acute ischaemic stroke secondary to large vessel occlusion (LVO) in the anterior circulation [1]. Following this, the American Heart/Stroke Association in their latest guideline gave a class I recommendation for EVT in patients with large artery occlusions (LVO) in the anterior circulation as an adjunct to intravenous alteplase [2]. In selected patients with contraindications to alteplase, direct EVT within $6 \mathrm{~h}$ of stroke onset [2] was given class IIa recommendation.

However, direct evidence for EVT in alteplase-ineligible patients is less compelling. Among the randomised controlled trials of endovascular therapy, only $5.7 \%$ of patients did not receive tPA, and there was substantial heterogeneity in the effect on of EVT with and without tPA on good functional outcome ( $\mathrm{mRS} \leq 2$ ) (EVT plus tPA: weight $94.3 \%$, risk ratio 1.43 , 95\% CI 1.23-1.61 vs. EVT without tPA: weight 5.7\%, risk ratio 2.47, 95\% CI 1.43-4.27; $I^{2}=74 \%, p$ heterogeneity 0.05 ] [1]. A randomised trial [3] designed to specifically investigate the efficacy and safety of EVT in patients ineligible for alteplase was suspended after recruiting a few patients.

It is also uncertain whether the treatment effect of EVT is so powerful in its own right that bridging alteplase may not add to the efficacy of EVT and may even compromise the safety of EVT by increasing the risks of bleeding [4].

We aimed to survey the proportion of patients suitable for EVT who are alteplase-ineligible and to compare the safety and effectiveness of standard care of acute large artery ischaemic stroke by EVT plus thrombolysis with that of EVT alone in a tertiary hospital clinical stroke service.

\section{Methods}

\section{Design and Setting}

We performed a retrospective analysis of acute ischaemic stroke patients treated with EVT at our centre. Sir Charles Gairdner Hospital is a tertiary hospital providing acute reperfusion treatment for patients with acute ischaemic stroke.

\section{Acute Stroke Assessment and Treatment Process}

Patients with suspected acute stroke are assessed at presentation by the stroke team comprising a stroke neurologist, interventional neuroradiologist, and stroke nurse. The stroke neurologist establishes the clinical diagnosis of stroke from history, clinical exami- 


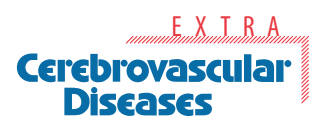

Fig. 1. Contraindications to intravenous thrombolysis $(n=29)$. Out of time ( $>4.5 \mathrm{~h}$ from onset), $n=6$ (wake-up strokes/uncertain onset, $n=5$ ); recent surgery (within 14 days), $n=5$; recent myocardial infarction (MI) (within 30 days), $n=2$; acute injury (during stroke ictus), $n=3$; therapeutic anticoagulation, $n=5$; previous intracranial haemorrhage, $n=2$; NIHSS too high ( $>25), n=2$; subacute infracts (on initial imaging), $n=1$; agitation (patient agitated/violent), $n=1$; previous gastrointestinal (GI) bleeding, $n=1$.

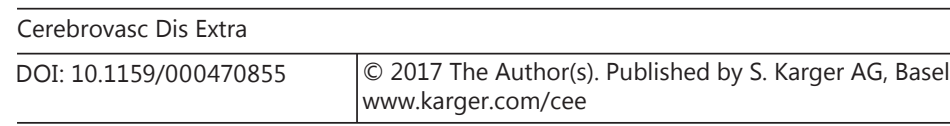

Wee et al.: Outcomes of Endovascular Thrombectomy at a Tertiary Stroke Centre

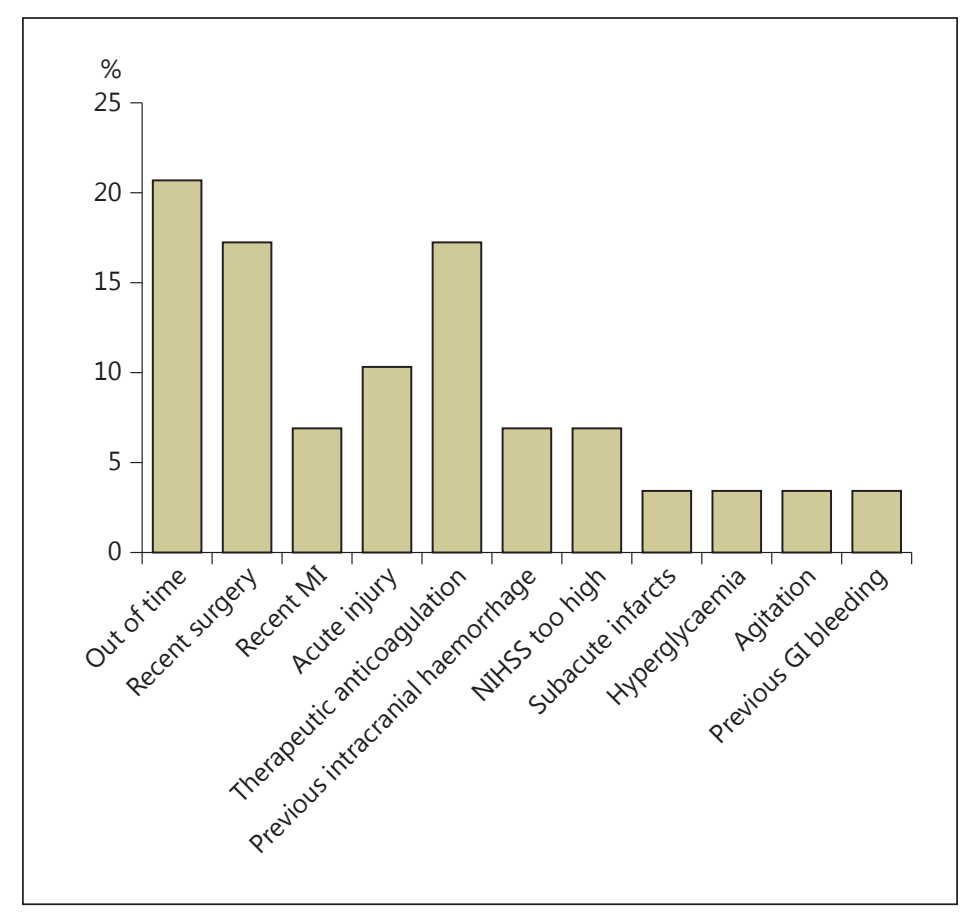

nation, and imaging findings. The severity of stroke is assessed by the National Institutes of Health Stroke Scale (NIHSS).

If the initial plain computed tomography (CT) scan excluded intracranial haemorrhage, a CT perfusion scan is undertaken and CT angiographic images of the intracranial vessels are extracted from the perfusion study.

Patients assessed within $4.5 \mathrm{~h}$ of stroke onset who have no contraindications to alteplase, including established hypodense infarcts involving >1/3 MCA territory, and who provided informed consent, are treated with intravenous alteplase according to a standard protocol of $0.9 \mathrm{mg} / \mathrm{kg} ; 10 \%$ is given as a bolus and the remaining $90 \%$ infused over $1 \mathrm{~h}$.

EVT is offered as adjunctive treatment to alteplase if the CT angiographic image identifies a LVO in the anterior circulation, and if the CT perfusion scan identifies a salvageable penumbra (increased time to peak) surrounding any core infarction (decreased cerebral blood volume) [5]. Patients proceed to EVT while the alteplase infusion is in progress.

Patients with contraindications to intravenous thrombolysis who are assessed as having a large artery anterior circulation ischaemic stroke within $6 \mathrm{~h}$ of onset (Fig. 1) were considered for EVT alone.

Consent for treatment with EVT and alteplase is obtained from the patient or next of kin.

For patients undergoing EVT, vascular access is obtained via the transfemoral approach using ultrasound guidance. An initial diagnostic angiogram utilising bi-plane high-resolution angiography is acquired. Proximal extracranial stenosis is stented if necessary to facilitate intracranial access and any thrombus removed by a combination of stent retrievers and direct aspiration systems.

Angiographic confirmation of reperfusion is obtained for all patients after the procedure and graded according to the modified Thrombolysis in Cerebral Infarction (mTICI) system [6], as follows:

- 0: No perfusion beyond the site of occlusion.

- 1: Penetration but not perfusion. Contrast penetration past the initial obstruction but with minimal filling of the normal territory. 
- 2: Incomplete perfusion. Contrast passes occlusion and opacifies the distal arterial bed, but the rate of clearance if slower or incomplete when compared to noninvolved territories.

- 2A: Some perfusion with the distal branch filling $<50 \%$ of the territory visualized.

- 2B: Substantial perfusion with the distal branch filling $\geq 50 \%$ of the territory visualized.

- 2C: Near-complete perfusion except for slow flow in a few distal cortical vessels, or presence of small distal cortical emboli.

- 3: Complete perfusion with normal filling of all distal branches.

A cone beam CT may be performed at the end of the procedure to check for haemorrhage. Repeat imaging with either CT or magnetic resonance imaging scan is performed on days 2-3 of admission and as clinically indicated.

Ascertainment and Analysis of Data

Patients assessed by the stroke team were prospectively recorded and entered into an acute stroke registry from 2007. We studied patients from October 2013 until April 2016. Some patients from February 2015 and prior were included in a separate state-wide audit published elsewhere [7]. Baseline information including age, sex, premorbid conditions, current medications, and initial NIHSS scores were obtained from case note review. Outcome measures included reperfusion grade according to the mTICI system, reperfusion time, and NIHSS score at $24 \mathrm{~h}$. Complete reperfusion was defined as mTICI 2B-3 on post-procedure angiography

Significant clinical improvement was defined as a NIHSS score improvement of $\geq 8$ compared to baseline, or a NIHSS score of $0-2$, at $24 \mathrm{~h}[8,9]$. As the NIHSS score was not routinely scored after the procedure, this was derived where there was sufficient information from clinical observations of the medical and allied health team. Patients who were in intensive care or too unwell to be accessed had no score. Patients with no NIHSS scores were adjudicated to not have significantly improved. Functional outcome at 3 months after ictus was not available for most patients as they were transferred to other departments for follow-up care.

Adverse outcomes include death at any time during current admission, subarachnoid haemorrhage and haemorrhagic conversion (parenchymal haematoma grades I and II by the ECASS [10] criteria) on post-procedure cone beam CT or repeat imaging on days 2-3, puncture site haematomas, and pseudoaneurysm on post-procedural review the following day. There were no cases of arterial dissection, frank vessel perforation, or systemic bleeding.

All statistical analyses were performed by IBM SPSS Statistics for Macintosh, version 24.0 (IBM Corp., Armonk, NY, USA). Differences between groups were assessed with the Pearson $\chi^{2}$ test for categorical variables and the Student $t$ test for continuous variables. Patient demographics, cardiovascular risks, and pre-existing medications were compared.

Approval for publication of this study was granted by the hospital's human research ethics committee.

\section{Results}

Sixty-two patients with acute ischaemic stroke received EVT between October 2013 and April 2016. Fifty patients with stroke secondary to LVO in the anterior circulation are described; 10 had strokes in the posterior circulation, while case notes for 2 patients were unavailable.

Among the 50 patients undergoing EVT, 21 received concurrent alteplase and 29 EVT alone. Contraindications to intravenous thrombolysis for patients who received EVT alone are shown in Figure 1. The baseline characteristics of the 21 patients undergoing EVT 
Table 1. Baseline characteristics and univariate analysis

\begin{tabular}{|c|c|c|c|}
\hline & $\begin{array}{l}\text { EVT + thrombolysis } \\
(n=21)\end{array}$ & $\begin{array}{l}\text { EVT alone } \\
(n=29)\end{array}$ & $p$ value \\
\hline \multicolumn{4}{|l|}{ Baseline characteristics } \\
\hline Mean age \pm SD, years & $73 \pm 16$ & $71 \pm 14$ & 0.745 \\
\hline Male sex & $8(38)$ & $16(55)$ & 0.233 \\
\hline Mean NIHSS score \pm SD & $15(5)$ & $15(7)$ & 0.780 \\
\hline \multicolumn{4}{|l|}{ Cardiovascular risk factors } \\
\hline Hypertension & $13(61.9)$ & $21(72.4)$ & 0.432 \\
\hline Atrial fibrillation & $9(42.9)$ & $17(58.6)$ & 0.271 \\
\hline Coronary heart disease & $4(19.0)$ & $9(31.0)$ & 0.340 \\
\hline Previous TIA/stroke & $5(23.8)$ & $3(10.3)$ & 0.255 \\
\hline Diabetes & $4(19.0)$ & $10(34.5)$ & 0.230 \\
\hline Hyperlipidaemia & $8(38.1)$ & $15(51.7)$ & 0.340 \\
\hline Smoking & $4(19.0)$ & $2(6.9)$ & 0.223 \\
\hline \multicolumn{4}{|l|}{ Pre-existing medications } \\
\hline Anti-platelets & $11(52.4)$ & $9(31.0)$ & 0.128 \\
\hline Warfarin & $1(4.8)$ & $3(10.3)$ & 0.630 \\
\hline DOAC & $0(0.0)$ & $3(10.3)$ & 0.254 \\
\hline Statin & $7(33.3)$ & $14(48.3)$ & 0.291 \\
\hline \multicolumn{4}{|l|}{ Vessel occluded } \\
\hline ICA & $5(23.8)$ & $6(21.7)$ & 0.616 \\
\hline M1 & $11(52.4)$ & $12(41.4)$ & \\
\hline M2 & $5(23.8)$ & $11(37.9)$ & \\
\hline Tandem extracranial occlusion & $4(19.0)$ & $3(10.3)$ & 0.434 \\
\hline \multicolumn{4}{|l|}{ Treatment } \\
\hline Mean time to initial imaging $\pm \mathrm{SD}$, h:min & $1: 42 \pm 0: 35(n=20)$ & $2: 50 \pm 2: 51$ & 0.091 \\
\hline Mean time to procedure start $\pm \mathrm{SD}$, h:min & $2: 45 \pm 1: 00$ & $3: 40 \pm 3: 07$ & 0.204 \\
\hline
\end{tabular}

Values are shown as $n(\%)$, unless otherwise indicated. EVT, endovascular thrombectomy; NIHSS, National Institutes of Health Stroke Scale; TIA, transient ischaemic attack.

combined with alteplase and the 29 with EVT alone were evenly matched, as shown in Table 1. The outcomes of the 21 patients undergoing EVT combined with alteplase and the 29 with EVT alone are shown in Table 2.

Reperfusion to mTICI grade 2B-3 was similar between EVT plus thrombolysis and EVT alone ( 90.5 vs. $93.1 \%, p=0.735$ ). Clinical improvement defined by NIHSS score improvement $\geq 8$ or NIHSS score $0-2$ at $24 \mathrm{~h}(47.6$ vs. $51.7 \%, p=0.774$ ) was also similar between the groups. Bridging alteplase was associated with a nonsignificant trend to more groin haematomas (23.8 vs. $10.3 \%$ ) and groin pseudoaneurysms (4.8 vs. $0 \%$ ). Intracranial haemorrhage rates were higher in patients receiving EVT alone ( 14.3 vs. $20.7 \%, p=0.716)$. Adverse outcomes which were higher in patients who received bridging thrombolysis include clot fragmentation ( 9.5 vs. $0 \%, p=0.171$ ) and inpatient demise ( 20 vs. $7.1 \%, p=0.184)$, both of which did not reach significance.

\section{Discussion}

In this study, more patients $(n=29 ; 58 \%)$ with acute ischaemic stroke and a proximal large anterior circulation artery, suitable for EVT, had a contraindication to alteplase, and were treated with EVT alone than patients $(n=21 ; 42 \%)$ who were treated conventionally, 
Table 2. Outcomes

\begin{tabular}{|c|c|c|c|}
\hline & $\begin{array}{l}\text { EVT + thrombolysis } \\
(n=21)\end{array}$ & $\begin{array}{l}\text { EVT alone } \\
(n=29)\end{array}$ & $\begin{array}{l}p \\
\text { value }\end{array}$ \\
\hline mTICI 2B-3 reperfusion & $19(90.5)$ & $27(93.1)$ & 0.735 \\
\hline Mean time to reperfusion $\pm \mathrm{SD}$, h:min & $3: 47 \pm 1: 12(n=19)$ & $4: 33 \pm 3: 08(n=29)$ & 0.322 \\
\hline Mean improvement in NIHSS score at $24 \mathrm{~h} \pm$ SD & $6.7 \pm 4.6(n=19)$ & $6.9 \pm 5.3(n=27)$ & 0.872 \\
\hline \multicolumn{4}{|l|}{ Significant improvement in NIHSS score $\geq 8$ or } \\
\hline $0-2$ at $24 \mathrm{~h}$ & $10(47.6)$ & $15(51.7)$ & 0.774 \\
\hline Clot lysis before EVT & $1(4.8)$ & $0(0)$ & 0.420 \\
\hline \multicolumn{4}{|l|}{ Complications } \\
\hline Groin haematoma & $5(23.8)$ & $3(10.3)$ & 0.170 \\
\hline Pseudoaneurysm & $1(4.8)$ & $0(0.0)$ & \\
\hline Intracranial haemorrhage $^{1}$ & $3(14.3)$ & $6(20.7)$ & 0.716 \\
\hline Subarachnoid haemorrhage & $0(0.0)$ & $2(6.9)$ & 0.503 \\
\hline Parenchymal haematoma & $2(9.5)$ & $2(6.9)$ & 1.000 \\
\hline Clot fragmentation and distal embolization & $2(9.5)$ & $0(0.0)$ & 0.171 \\
\hline Recurrent stroke & $1(5.0)$ & $1(3.6)$ & 0.807 \\
\hline Inpatient demise & $4(19.0)$ & $2(6.9)$ & 0.223 \\
\hline
\end{tabular}

according to current guidelines, with alteplase and EVT. Although the numbers are small, and estimates imprecise, the data suggest that alteplase ineligibility is not uncommon.

Overall, approximately half of the patients in our series who underwent EVT showed significant clinical improvement at $24 \mathrm{~h}$ (47.6\% for EVT plus thrombolysis vs. $51.7 \%$ for EVT alone, $p=0.774$ ). Employing criteria similar to REVASCAT [9] of including only patients with NIHSS score $\geq 6$ who received arteriotomy before $8 \mathrm{~h}$, and excluding patients with no NIHSS scores at $24 \mathrm{~h}$ (from early death or intubation), we have a subgroup of 40 patients. Of these, $52.5 \%$ showed significant early neurological improvement $(52.6 \%$ for EVT plus thrombolysis vs. $52.4 \%$ for EVT alone, $p=0.987$ ). These results are comparable to the $59 \%$ of patients showing early neurological improvement in REVASCAT and confirm our earlier findings [7] that results from the EVT trials are achievable in a service setting.

Although the mean time to reperfusion was nonsignificantly increased in direct EVT patients ( $3 \mathrm{~h} 47 \mathrm{~min}$ in EVT plus thrombolysis vs. $4 \mathrm{~h} 33 \mathrm{~min}$ in EVT alone, $p=0.322$ ), outcomes were similar between the 2 groups. This is similar to prior hospital-based series [11-13] and adds to the literature supporting the use of direct EVT in patients ineligible for alteplase. We also note that, unlike in the 5 trials where only $14.7 \%(188$ of 1,278) of the patients were ineligible for alteplase [14], the proportion of such patients in our study was 58\% (29 of 50). If corroborated in other series, this finding suggests that on a population basis, greater benefits may be derived from treating these patients who had less representation and evidence from randomised trials.

Complication rates of parenchymal haematomas (9.5\%) and patient demise (19.0\%) are within the ranges from the trials $(5-11 \%[8,9,15,16]$ and $9-18.9 \%[8,9,15-17]$, respectively.

Contrary to our hypothesis, and the earlier series by Broeg-Morvay et al. [11], the rates of intracranial haemorrhage were higher in patients who did not receive alteplase, albeit nonsignificantly. This was mainly due to subarachnoid haemorrhage ( 0 vs. $7.1 \%, p=0.504$ ) which occurred in 2 patients with only trace amounts of blood (grade 2 on the Fisher scale) 
[18]. There was no angiographic evidence of arterial rupture or contrast extravasation. Both patients were conservatively managed and did not require any additional intervention. Similar cases of subarachnoid haemorrhage without angiographic evidence of vessel injury were previously reported by Yoon et al. [19]. This was seen in 16.2\% (12 of 74) of patients receiving mechanical thrombectomy for acute ischaemic stroke in that series. Significantly, these patients showed no post-procedural neurological deterioration and had similar clinical outcomes to those without subarachnoid haemorrhage, suggesting a relatively benign outcome for such cases. Aetiology remains uncertain, however, and with small numbers in published literature, more studies are needed.

Mortality was nonsignificantly higher in patients who received alteplase (20 vs. $7.1 \%$, $p=0.184$ ). Though this also did not reach statistical significance, it is consistent with the series mentioned above.

We also noticed cases of clot fragmentation and distal embolization in the bridging thrombolysis group ( 9.5 vs. $0 \%, p=0.171$ ). Again, though statistics were limited by the sample size, this was significant as the thrombus became inaccessible and patients failed to reperfuse. On the other hand, complete clot lysis was seen in $4.8 \%$ of patients with bridging versus $0 \%$ of patients with EVT alone $(p=0.420)$. In a single case of a patient receiving intravenous thrombolysis, partial dissolution of the thrombus was observed during the diagnostic angiogram. This completely lysed upon direct instillation of $3 \mathrm{mg}$ of alteplase to the clot. This novel finding suggests that bridging thrombolysis before EVT may not be beneficial for some patients and requires further study.

The strengths of this study are that the patients are from a single centre and were managed consistently by a dedicated group of stroke neurologists and neurointerventionalists. Recruitment was consecutive, and real-world outcomes are showed. The main limitation is the small sample size and lack of follow-up data.

In conclusion, our study supports the use of direct endovascular clot retrieval in patients in whom alteplase is contraindicated. We observed no additional benefit of adding intravenous thrombolysis to EVT for outcomes of reperfusion and neurological impairment at $24 \mathrm{~h}$, but the estimates are imprecise due to small numbers. Nevertheless, the results are consistent with observational data from other endovascular centres and support a randomised controlled trial comparing the safety and efficacy of proceeding directly to endovascular clot retrieval against the current guidelines to proceed to endovascular clot retrieval with bridging thrombolysis in patients with acute ischaemic stroke due to LVO in the anterior circulation.

\section{References}

1 Rodrigues FB, Neves JB, Caldeira D, Ferro JM, Ferreira JJ, Costa J: Endovascular treatment versus medical care alone for ischaemic stroke: systematic review and meta-analysis. BMJ 2016;353:i1754.

$\longrightarrow 2$ Powers WJ, Derdeyn CP, Biller J, Coffey CS, Hoh BL, Jauch EC, et al: 2015 American Heart Association/American Stroke Association Focused Update of the 2013 Guidelines for the Early Management of Patients with Acute Ischemic Stroke Regarding Endovascular Treatment: A Guideline for Healthcare Professionals from the American Heart Association/American Stroke Association. Stroke 2015;46:3020-3035.

-3 Bendszus M, Thomalla G, Knauth M, Hacke W, Bonekamp S, Fiehler J: Thrombectomy in patients ineligible for iv tPA (THRILL). Int J Stroke 2015;10:950-955.

-4 Grotta JC, Hacke W: Stroke neurologist's perspective on the new endovascular trials. Stroke J Cereb Circ 2015;46:1447-1452.

5 Chiu AH, Phillips TJ, Phatouros CC, Singh TP, Hankey GJ, Blacker DJ, et al: CT perfusion in acute stroke calls: A pictorial review and differential diagnoses. J Med Imaging Radiat Oncol 2016;60:165-171.

-6 Almekhlafi MA, Mishra S, Desai JA, Nambiar V, Volny 0, Goel A, et al: Not all "successful" angiographic reperfusion patients are an equal validation of a modified TICI scoring system. Interv Neuroradiol 2014;20:21-27.

7 Kabra R, Phillips TJ, Saw J-L, Phatouros CC, Singh TP, Hankey GJ, et al: Mechanical thrombectomy for anterior circulation stroke: 5-year experience in a statewide service with differences in pretreatment time metrics across two hospitals sites. J Neurointerv Surg 2016, Epub ahead of print. 
8 Campbell BCV, Mitchell PJ, Kleinig TJ, Dewey HM, Churilov L, Yassi N, et al: Endovascular therapy for ischemic stroke with perfusion-imaging selection. N Engl J Med 2015;372:1009-1018.

-9 Jovin TG, Chamorro A, Cobo E, de Miquel MA, Molina CA, Rovira A, et al: Thrombectomy within 8 hours after symptom onset in ischemic stroke. N Engl J Med 2015;372:2296-2306.

10 Hacke W, Kaste M, Fieschi C, Toni D, Lesaffre E, von Kummer R, et al: Intravenous thrombolysis with recombinant tissue plasminogen activator for acute hemispheric stroke. The European Cooperative Acute Stroke Study (ECASS). JAMA 1995;274:1017-1025.

11 Broeg-Morvay A, Mordasini P, Bernasconi C, Bühlmann M, Pult F, Arnold M, et al: Direct mechanical intervention versus combined intravenous and mechanical intervention in large artery anterior circulation stroke: a matched-pairs analysis. Stroke 2016;47:1037-1044.

12 Rebello LC, Haussen DC, Grossberg JA, Belagaje S, Lima A, Anderson A, et al: Early endovascular treatment in intravenous tissue plasminogen activator-ineligible patients. Stroke 2016;47:1131-1134.

13 Weber R, Nordmeyer H, Hadisurya J, Heddier M, Stauder M, Stracke P, et al: Comparison of outcome and interventional complication rate in patients with acute stroke treated with mechanical thrombectomy with and without bridging thrombolysis. J Neurointerv Surg 2017;9:229-233.

14 Goyal M, Menon BK, van Zwam WH, Dippel DWJ, Mitchell PJ, Demchuk AM, et al: Endovascular thrombectomy after large-vessel ischaemic stroke: a meta-analysis of individual patient data from five randomised trials. Lancet 2016;387:1723-1731.

15 Berkhemer OA, Fransen PSS, Beumer D, van den Berg LA, Lingsma HF, Yoo AJ, et al: A randomized trial of intraarterial treatment for acute ischemic stroke. N Engl J Med 2015;372:11-20.

16 Saver JL, Goyal M, Bonafe A, Diener H-C, Levy EI, Pereira VM, et al: Stent-retriever thrombectomy after intravenous t-PA vs. t-PA alone in stroke. N Engl J Med 2015;372:2285-2295.

17 Goyal M, Demchuk AM, Menon BK, Eesa M, Rempel JL, Thornton J, et al: Randomized assessment of rapid endovascular treatment of ischemic stroke. N Engl J Med 2015;372:1019-1030.

18 Fisher CM, Kistler JP, Davis JM: Relation of cerebral vasospasm to subarachnoid hemorrhage visualized by computerized tomographic scanning. Neurosurgery 1980;6:1-9.

19 Yoon W, Jung MY, Jung SH, Park MS, Kim JT, Kang HK: Subarachnoid hemorrhage in a multimodal approach heavily weighted toward mechanical thrombectomy with solitaire stent in acute stroke. Stroke 2013;44:414419. 